\title{
The evolutionary status of EK Cephei: rotating and standard models
}

\begin{abstract}
A. Claret
Instituto de Astrofísica de Andalucía, CSIC, Apartado 3004, 18080 Granada, Spain

e-mail: claret@iaa.es

Received 28 March 2005 / Accepted 12 September 2005

\section{ABSTRACT}

The evolutionary status of the double-lined eclipsing binary EK Cep is reanalysed. Due to problems with the empirical determination of its effective temperatures, we have used a different approach to compare the observations with theoretical models: we have adopted the effective temperature ratio, which is a better determined parameter from light curve analysis, instead of their absolute values. Such an approach is supported observationally by the inconsistency found in the photometric distances for both components of EK Cep. By using the effective temperature ratio, masses, radii, apsidal-motion rate and lithium depletion, we have found that standard models are able to fit the physical properties of EK Cep simultaneously at the same isochrone. In the case of rotating models, we have also found acceptable solutions without the need for a very fast rotating core. On the other hand, the apsidal-motion rate of EK Cep is critically revised.
\end{abstract}

Key words. stars: binaries: eclipsing - stars: evolution - stars: rotation

\section{Introduction}

The eclipsing binary system EK Cephei is a very interesting object to test evolutionary stellar models due to its intrinsic characteristics: 1) it is a double-lined eclipsing binary with accurate determination of absolute dimensions, at least concerning masses and radii; 2) its secondary component is a Pre MainSequence star (PMS); 3) the system presents apsidal motion with a high relativistic contribution; 4) lithium has been measured for the less massive mate and 5) an evaluation of the metallicity of the binary is also available. Often, double-lined eclipsing binaries are good candidates to probe the capability of stellar models but never with as high a number of constraints as in the case of EK Cep.

Among the characteristics of EK Cep (Table 1, see Popper 1987 and Andersen 1991), the most important is probably the PMS nature of the secondary since it presents low surface gravity (Popper 1987). This was confirmed later by the lithium determination obtained by Martín \& Rebolo (1993). The theoretical interpretation of EK Cep is thus a tractable task. Indeed, in 1995 Claret et al. made the first attempt to explain the evolutionary status, the apsidal-motion and the lithium depletion of this binary star. They confirmed the secondary as a PMS star while the more massive companion seems to be at the begining of the hydrogen- burning phase. The theoretical apsidal-motion rate was found to be in good agreement with the observational data. The level of lithium depletion was also found to be in agreement with the observations of the secondary while for the primary Claret et al. (1995) reported no significant depletion.
Table 1. Absolute dimensions of EK Cep (solar, cgs, K units).

\begin{tabular}{lll}
\hline \hline & Primary & Secondary \\
Mass & $2.029 \pm 0.023$ & $1.124 \pm 0.012$ \\
Radius & $1.579 \pm 0.007$ & $1.315 \pm 0.006$ \\
$\log g$ & $4.349 \pm 0.010$ & $4.251 \pm 0.006$ \\
$\log L$ & $1.17 \pm 0.04$ & $0.19 \pm 0.07$ \\
$\log T_{\text {eff }}$ & $3.954 \pm 0.010$ & $3.756 \pm 0.015$ \\
\hline
\end{tabular}

The radii of both components were approximately fitted at a common age of $20 \times 10^{6}$ years. To avoid problems with effective temperature calibration, only isochrones in the masses $\times$ radii plane were used.

More recently, Yildiz (2003) also analysed the system. Some of his results will be discussed in the next sections. Marques et al. (2004) used a $\chi^{2}$ minimization method to find the best theoretical models. They were not able, however, to reproduce the radii for both components simultaneosly for the same isochrone. Given that the evolutionary scenario is neither clear nor satisfactory, we decided to reanalyse EK Cep using a new version of our evolution code (Claret 2004) incorporating new input physics and a different approach to the problem.

\section{The effective temperatures of EK Cep}

Our procedure to explain the evolutionary status of EK Cep is different from that by Yildiz (2003) and by Marques et al. (2004) with regards to the observed constraints to be used. The effective temperatures of EK Cep and their respective 


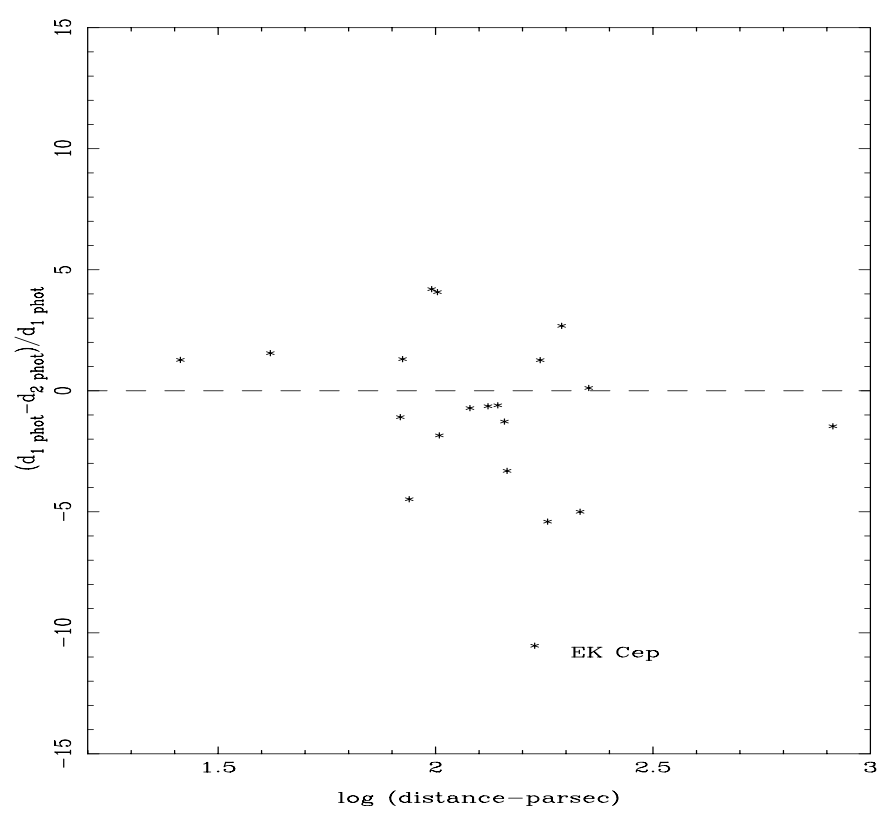

Fig. 1. Percent differences between the photometric distances for the individual components of detached eclipsing binaries. Note the position of EK Cep. The data are taken from Ribas et al. (1998).

error bars are key to model the system and the mentioned authors used them as strong constraints to their solutions. However, in general, effective temperatures are not a direct measurement: they depend on a previous calibration, based on stellar atmosphere models. The involved uncertainties are often larger than we obtain simply by interpolating in the atmosphere grids. If we consider the uncertainties in the opacities, the plane paralell approach and the adopted extintion law, we conclude that, in some cases, the error estimation is too low. In the particular case of EK Cep the situation is even worse: the mass ratio is around 0.5 , and as a consequence there is an intrinsic difficulty in the determination of the effective temperature for the less massive component, which is around 10 times fainter than its mate.

To reinforce those difficulties we plot in Fig. 1 the relative differences between the individual photometric distances for some detached eclipsing binaries taken from the work by Ribas et al. (1998). The inconsistency found for both components of EK Cep is notorious and the distances obtained for each individual component differ by around $10 \%$. This is the largest discrepancy found in the sample. This may indicate some problems with the absolute dimensions of this binary (and with other systems too). The radii are often well measured parameters in detached eclipsing binaries and do not depend on previous calibrations. The same does not apply to the effective temperatures. As already mentioned, the effective temperatures are strongly dependent on calibrations. In the case of EK Cep this limitation may be even larger given the PMS nature of the secondary because the calibration of PMS stars does not have similar observational/theoretical support as for normal mainsequence stars. Popper (1987) reported some anomalies in the secondary of EK Cep, such as an excess of radiation in the $\mathrm{B}$ band or discrepancies between $(B-V)$ and $(V-R)$.
The determination of the effective temperature of the primary also presents similar problems: $T_{\text {eff }}$ (photometry) is about $7 \%$ larger than $T_{\text {eff }}$ (Hipparcos). From these results we conclude that the effective temperatures are not, in this case, a good selection to constrain the theoretical models. In fact, the difficulties with the effective temperatures seem to be a common general trend: eclipsing binaries present the largest internal scattering when used as distance indicators, as pointed out by Alves (2004). This indicates that a serious and systematic problem may be present in the determination of the effective temperatures of such stars. However, even if the effective temperatures are not well determined (mainly concerning the error bars) they still can be used to constrain the models for EK Cep. We decided to use the effective temperature ratio $T R=T_{\text {eff } 2} / T_{\text {eff1 } 1}$, instead of their absolute values, since this ratio is determined much more accurately from the light curve analysis and is free from calibrations.

\section{Alternative models for EK Cep}

In Claret et al. (1995) we did not directly use the effective temperatures in order to avoid problems with calibrations and an acceptable solution was found for $(X, Z)=(0.705,0.015)$ using the observed masses and radii as contraints. However, in the past 10 years, the input physics of our code of stellar evolution has changed drastically: the opacities, the nuclear network, the mass loss rates and the mass range for which the models are computed. For radiative opacities we have adopted the calculations by Iglesias \& Rogers (1996) completed with Alexander \& Ferguson (1994) results for lower temperatures. A more elaborated nuclear network with up to 47 isotopes can also be used for the production/destruction of light elements or when more advanced stages of nucleosynthesis are required. The Sun was calibrated by using $(X, Z, \alpha)=(0.686,0.02,1.68)$. For a more detailed description of the code, see Claret (2004). For the present study, in order to guarantee more precise calculations we have restricted the triangle used to define an envelope in the HR diagram to $\Delta \log T_{\text {eff }}=0.001$ and $\Delta \log L=0.004$ or smaller.

\subsection{Non-rotating models}

Before computing the stellar models for both components of EK Cep it is crucial to define clearly which observational parameters will be used as constraints. Here we shall adopt the masses, the radii and the effective temperature ratio, as explained above. Also, we will restrict models around $Z \approx 0.02$, as given by Martín \& Rebolo (1993). The apsidal-motion and the lithium depletion will be treated after obtaining an acceptable solution. A key question is whether we should use rotating or non-rotating models. Given that all stars rotate, rotating models should be preferred. However, the effects of rotation do not seem to be important for the present evolutionary status of EK Cep (given that no significant oblateness in the light curves is observed (Hill \& Ebbighausen 1984) which implies that the components are well within their Roche lobes and they are not fast rotators). Moreover, it will be interesting to explore the 

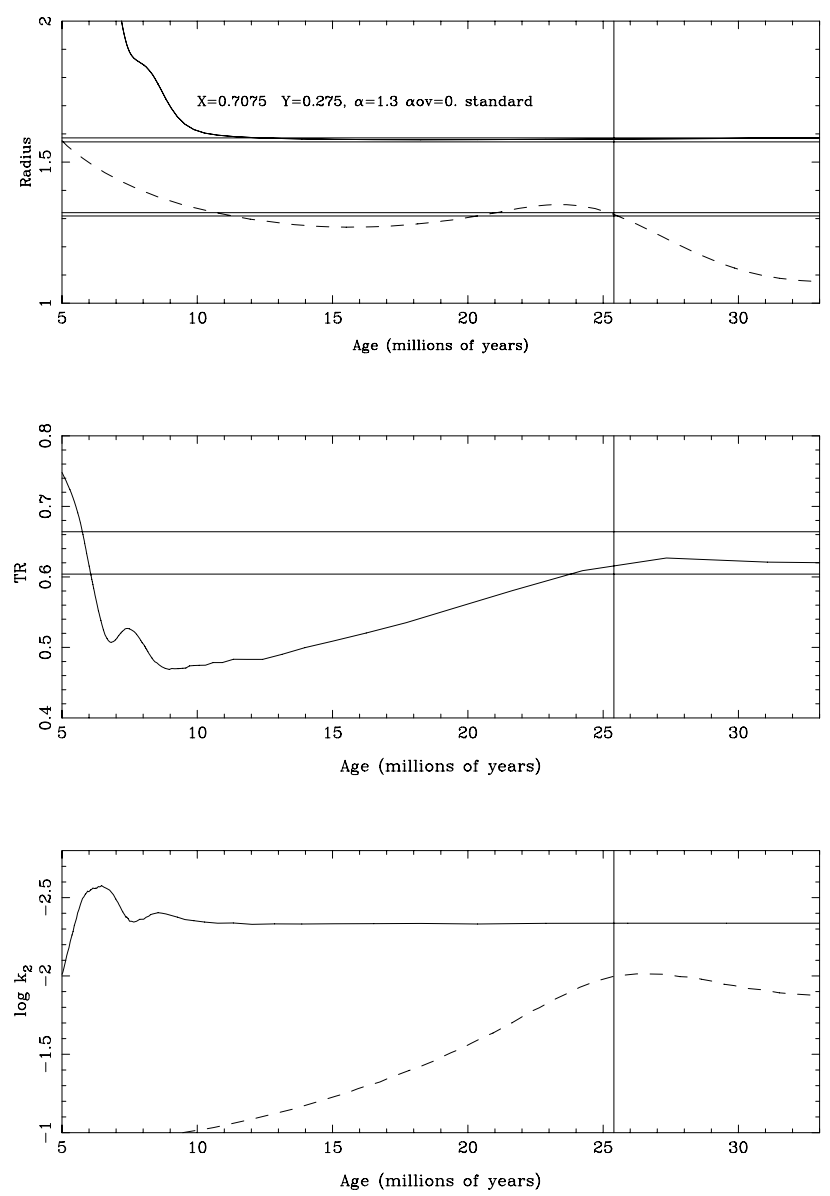

Fig. 2. Theoretical standard models for EK Cep (without rotation). The upper panel shows the radius of the models as a function of the age (continuous line represents the primary and dashed line denotes the secondary). The middle panel shows the effective temperature ratio TR. The bottom panel presents the apsidal-motion constants for EK Cep. Note that there is an acceptable agreement between radii and TR for a same isochrone (vertical lines). The horizontal lines represent the error bars.

capability of standard models to predict the physical properties of EK Cep.

As already mentioned, the primary component is at the begining of the hydrogen-burning phase. At this stage, stars evolve with almost constant radius. This makes it difficult to determine the stellar age. For both components of EK Cep we have found that, for $(X, Z)=(0.7075,0.0175)$, a mixing-length parameter $\alpha=1.3$ and no core overshooting (because its effects are not important here), the radii and the effective temperature ratio TR are on the same isochrone (Fig. 2). We have used a modified $\chi^{2}$ method to obtain the present solution. The derived age is around $25.4 \times 10^{6}$ years, a value larger than that derived in the 1995 paper. The fitting is acceptable and within the uncertainties. Similar solutions - or more refined ones - may be found by varying slightly the input physics of the models. The relative success of the theoretical predictions are not only due to the models. The selection of the observational parameters used to constrain the solutions was a key piece of information.

We do not consider the solution shown in Fig. 2 as a definitive one. However, standard models are also capable of

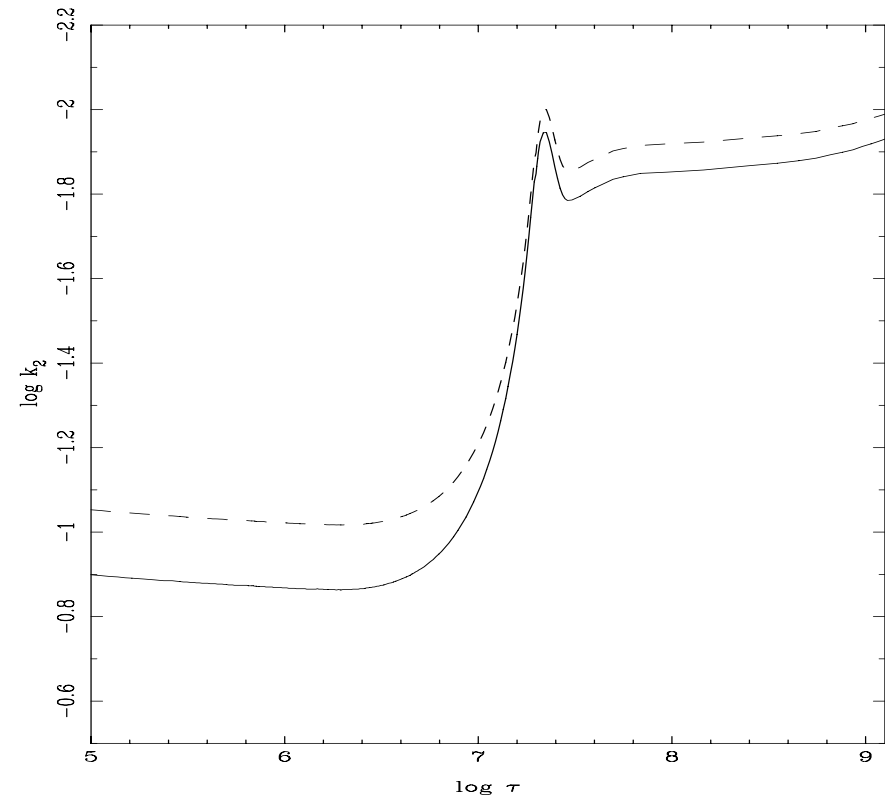

Fig. 3. Apsidal-motion constant $k_{2}$ as a function of time for a $1.12 M_{\odot}$ model. The model was followed from the PMS stage up to the giant branch. Continuous line represents $k_{2}$ as computed by integrating Radau differential equation while dashed one denotes calculations performed with Kopal approach. During the PMS phase, the differences can reach more than 0.20 dex.

predicting the absolute dimensions of EK Cep without the need to introduce a very fast rotating core (with all its problems discussed in the next section). Within this approach, let us investigate the apsidal-motion rate. For clarity, we write down the basic equations, say, the Radau differential equation:

$\frac{a \mathrm{~d} \eta_{j}}{\mathrm{~d} a}+\frac{6 \rho(a)}{\bar{\rho}(a)}\left(\eta_{j}+1\right)+\eta_{j}\left(\eta_{j}-1\right)=j(j+1)$

where

$\eta \equiv \frac{a}{\epsilon_{i}} \frac{\mathrm{d} \epsilon_{i}}{\mathrm{~d} a}$

and the relationship between $a$ and $r$ is

$r=a\left(1+\sum_{j=0}^{n} \epsilon_{j}(a) P_{j}(\theta)\right)$

where $a$ is the mean radius of a given equipotential, $\rho(a)$ is the mass density at the distance $a$ from the center, $\bar{\rho}(a)$ is the mean mass density within a sphere of radius $a, P_{j}(\theta)$ are the Legendre polynomials and $\epsilon_{j}$ describes the amplitude of the distortions. The $k_{j}$ are computed using the equation

$k_{j}=\frac{j+1-\eta_{j}(R)}{2\left(j+\eta_{j}(R)\right)}$

where $\eta_{j}(R)$ is the value at the surface of the model. Because the contributions for $j=3$ and 4 are small, we retain only the term with $j=2$.

Yildiz (2003) used the Kopal (1978) approximation to obtain the theoretical $k_{2}$. The approach is a very important point 
that restricts his analysis. Some time ago, Claret \& Giménez (1989) showed that if the Kopal approximation is used, instead of the appropriate Radau differential equation, a systematic deviation is detected. Since Kopal assumed a priori that $\frac{\rho}{\bar{\rho}} \gg 1$ - where $\rho$ is the local density and $\bar{\rho}$ is the mean density of a given stellar configuration - the values of $k_{2}$ obtained using such an approximation will be systematically smaller than those derived from the Radau equation, i.e., the Kopal approach gives more centrally condensed configurations. In Fig. 3 these effects can be seen. The continuous line represents the apsidalmotion constant $k_{2}$ for a standard model for EK CepB by integrating the Radau differential equation, and the dashed one that computed following the Kopal approach. The differences can be as high as 0.20 dex. The actual level of refinement in the apsidal-motion investigation is almost a order of magnitud better than these differences (Claret \& Willems 2002).

Let us evaluate the theoretical weighted $\bar{k}_{2}$ which will be useful to compare with the observational one:

$\bar{k}_{2 \text { theo }}=\frac{c_{21} k_{21 \text { theo }}+c_{22} k_{22 \text { theo }}}{c_{21}+c_{22}}$

where

$c_{2 i}=\left[\left(\frac{\Omega_{i}}{\Omega_{K}}\right)^{2}\left(1+\frac{m_{3-i}}{m_{i}}\right) f(e)+\frac{15 m_{3-i}}{m_{i}} g(e)\right]\left(\frac{R_{i}}{A}\right)^{5}$

$R_{i}$ is the stellar radius, $A$ the semi-major axis, $\Omega_{i}$ is the angular velocity of the component $i, e$ is the eccentricity and $\Omega_{K}$ is the Keplerian angular velocity. The functions $f(e)$ and $g(e)$ are given by

$f(e)=\left(1-e^{2}\right)^{-2}$

$g(e)=\frac{\left(8+12 e^{2}+e^{4}\right) f(e)^{2.5}}{8}$.

Introducing the observational data in the above equations, we have $c_{21}=8.91 \times 10^{-5} \pm 3.2 \times 10^{-6}$ and $c_{22}=9.47 \times 10^{-5} \pm 3.5 \times$ $10^{-6}$. Note that in order to compute $c_{2 i}$ we used the observed rotational velocities; synchronization or pseudo-synchonization were not used as default. For typical values of $k_{2}$ (Figs. 2, 4 and 5) it is easy to show that the contribution of the secondary to the classical apsidal-motion rate is larger than that for the primary although it is not dominant. The contribution of the primary is around $30 \%$ of the total.

The observed $k_{2}$ is related to the observed apsidal-motion rate:

$\bar{k}_{2 \mathrm{obs}}=\frac{1}{360\left(c_{21}+c_{22}\right)} \dot{\omega}_{\mathrm{obs}}$

where $\bar{k}_{2 \text { obs }}$ is the observed apsidal-motion constant to be compared with its theoretical value, given by Eq. (5). Before such a comparison, the relativistic contribution must be evaluated following Levi-Civita (1937)

$$
\frac{P}{U^{\prime}}=6.35 \times 10^{-6} \frac{\left(m_{1}+m_{2}\right)}{A\left(1-e^{2}\right)} .
$$

After the relativistic correction, we have $\log \bar{k}_{2 \mathrm{obs}}=-2.09 \pm$ 0.09. To obtain the observed rate of apsidal-motion we have
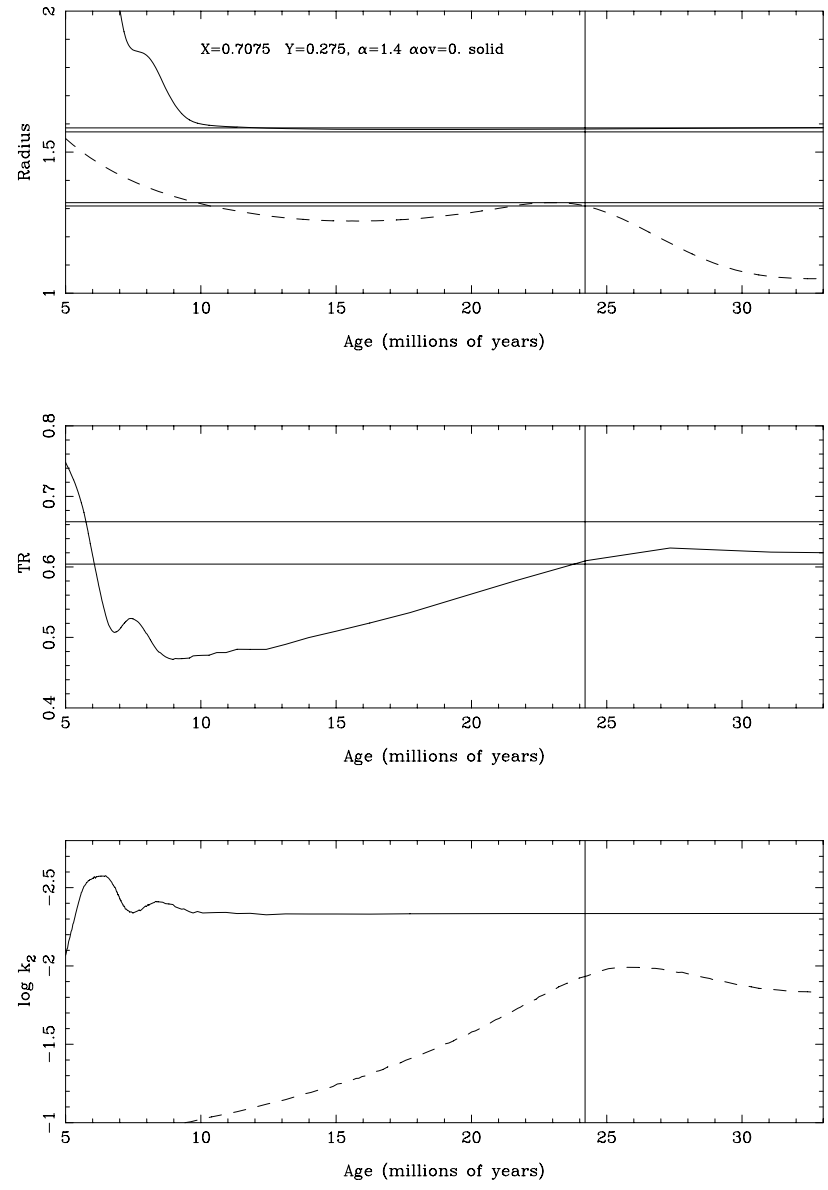

Fig. 4. The same as for Fig. 2 but taking into account solid rotation.

used the works by Khaliullin (1983), Giménez \& Margrave (1985), Hill \& Ebbighausen (1984), Caton et al. (1989), Diethelm (1992), Caton \& Burns (1993), Diethelm (1993), Lacy \& Fox (1994), Pajdosz (1993), Claret et al. (1995), Yildiz (2003, and references therein). The weighted least squares iterative method proposed by Giménez \& Bastero (1995) was used to derive the apsidal-motion rate from those works. By inferring the individual values of $k_{2}$ from Fig. 2 and applying Eq. (1) we will have $\log \bar{k}_{2 \text { theo }}=-2.10 \pm 0.06$. This can be considered as a good agreement and lies within the theoretical-observational errors.

Although the observed lithium depletion was not measured with high accuracy, we obtained for the secondary a depletion of $0.15 \mathrm{dex}$, approximately the same amount derived in the 1995 paper, which is within the uncertainty limits given by Martín \& Rebolo (1993).

\subsection{Rotating models}

The method used here to simulate rotation differs from that used by Yildiz (2003) mainly concerning how the differential equations are modified. A more detailed description and numerical details can be found in Kippenhahn \& Thomas (1970), Endal \& Sofia (1976) and Claret (1999). We restricted the 

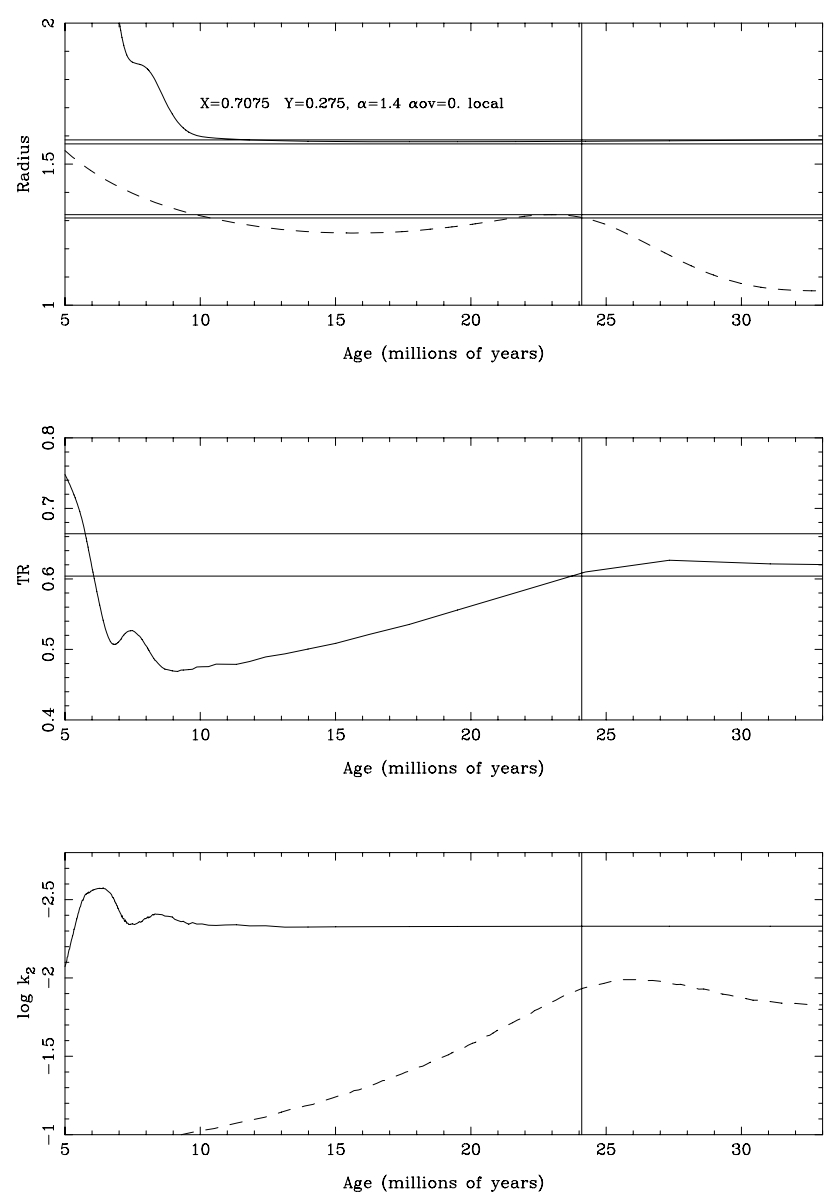

Fig. 5. The same as for Fig. 2 but taking into account rotation with local conservation of the angular momentum.

calculation to first order theory. Thus the total potential can be written (Kopal 1959)

$$
\begin{aligned}
\psi= & \frac{G M_{\psi}}{r}+\frac{1}{2} \Omega^{2} r^{2} \sin ^{2} \theta-\frac{4 \pi G}{5 r^{3}} P_{2}(\cos \theta) \\
& \times \int_{0}^{a} \rho \frac{\mathrm{d}}{\mathrm{d} a^{\prime}}\left(a^{\prime 5} f_{2}\right) \mathrm{d} a^{\prime}
\end{aligned}
$$

where

$r=a\left(1-f_{2} P_{2}(\cos \theta)\right)$

$f_{2}=\frac{5 \Omega^{2} a^{3}}{3 G M_{\psi}\left(2+\eta_{2}\right)}$

$\Omega$ is the angular velocity, $P_{2}(\cos \theta)$ is the second Legendre polynomial, $a$ the radius of the level surface, $\eta_{2}$ is connected with Radau's equation (Eq. (2)), and the remaining symbols have their usual meaning.

The differential equations of the structure are changed as follows:

$$
\begin{aligned}
\frac{\partial r_{\psi}}{\partial M_{\psi}} & =\frac{1}{4 \pi \rho r_{\psi}^{2}} \\
\frac{\partial P_{\psi}}{\partial M_{\psi}} & =-\frac{G M_{\psi}}{4 \pi r_{\psi}^{4}} f_{P}
\end{aligned}
$$

$\frac{\partial L_{\psi}}{\partial M_{\psi}}=\epsilon-T \frac{\partial S}{\partial t}$

$\frac{\partial \ln T_{\psi}}{\partial \ln P_{\psi}}=\frac{3 \kappa L_{\psi} P_{\psi} f_{T}}{16 \pi a c G M_{\psi} T_{\psi}^{4} f_{P}}$

and the Schwarzschild criterion is given by:

$\frac{\partial \ln T_{\psi}}{\partial \ln P_{\psi}}=\min \left[\nabla_{\mathrm{ad}}, \nabla_{\mathrm{rad}} \frac{f_{T}}{f_{P}}\right]$

where $\nabla_{\text {ad }}$ and $\nabla_{\text {rad }}$ are the spherical adiabatic and radiative gradients. The atmospheric factor $\mathrm{PGM} /\left(\kappa R^{2} \tau\right)$ is also changed by $f_{P}$, which is considered constant for the whole atmosphere and equal to that of the last point of the envelope. On the other hand, the factors $f_{P}$ and $f_{T}$ are given by

$f_{P}=4 \pi r_{\psi}^{4} \frac{1}{G M_{\psi} S_{\psi} \overline{g^{-1}}}$

and

$f_{T}=\left(\frac{4 \pi r_{\psi}^{2}}{S_{\psi}}\right)^{2} \frac{1}{\bar{g} \overline{g^{-1}}}$

where the mean values of $g$ and $g^{-1}$ are taken over equipotentials.

To compute $f_{P}$ and $f_{T}$ one has to know the relationship between $a$ and $r_{\psi}$. The radius of a sphere with equivalent volume is related to the radius of the level surface by

$r_{\psi}^{3}=a^{3}\left(1+\frac{3}{5} f_{2}^{2}-\frac{2}{35} f_{2}^{3}\right)$

The value of $a$ is obtained through iteration of Eq. (21).

Recently Yildiz (2003) proposed that rotating models could explain the physical properties of EK Cep. In that paper he concluded that only if the primary presents a rotating rapid core, can an acceptable solution be found. However, his method to introduce rotation may be not adequate because the only differential equation of the stellar structure which is changed is the hydrostatic one (Kippenhahn et al. 1970). Using such an approximation the oblatness effects are neglected.

Here we define the laws of angular momentum conservation to be adopted. We are implementing the fourth order differential equation which governs the evolution of the angular velocity (see for example, Zahn 1992) but the results are still preliminary and will not be considered here. We shall select two laws: 1) solid body rotation and 2) local conservation of angular momentum. Such laws are limits since for the first one the redistribution of the angular momentum is complete while for the second there is no redistribution. A more realistic treatment is expected to give intermediate results. Thus, both laws will serve as a good control for the calculations. We note that the transport of angular momentum due to rotationally-induced instabilities will not be considered because the related diffusion equation is coupled to the differential equation for the evolution of the angular velocity.

Case 1) The calculations of rotating models were carried out by introducing some numerical tolerances (for example, the 
number of shells was larger than 4000) in order to guarantee the conservation of the angular momentum. In order to get the rotating model displaying the observed rotational velocity for each component of EK Cep we have used trial models until the equatorial velocity is the same as that observed for both components. As EK Cep is not a very distorted system, the effects of rotation are expected to be not large. The factors $f_{P}$ and $f_{T}$ at the surface are not very different from 1 . We have used the same input physics (no core overshooting, no mass loss, etc.) as that for the standard models except for the mixing-length parameter $(\alpha=1.4)$. The agreement can be considered as good (Fig. 4) and the radii and effective temperature ratio can be fitted by the same isochone simultaneously $\left(24.2 \times 10^{6}\right.$ years $)$. Note that other solutions are also possible but they were not taken into account because the effective temperature ratio requirement was not fulfilled. Solid body rotation, although it is a very simple approach, is capable of predicting the properties of EK Cep without need for a rapidly rotating core. Moreover, as we will see below, it is not fully incompatible with the results from helioseismology. The theoretical individual values of $k_{2}$ inferred from the last panel of Fig. 4 give, according Eq. (1), $\log \bar{k}_{2 \text { theo }}=-2.10 \pm 0.06$, which is in agreement with observations $\left(\log \bar{k}_{2 \mathrm{obs}}=2.09 \pm 0.09\right)$. The depletion of lithium in the secondary component is of the same order as that for nonrotating models and also is in agreement with the observational data and their respective uncertainties.

Case 2) Other series of models were computed by assuming local conservation of the angular momentum. The results can be seen in Fig. 5. By inferring the values of $k_{2}$ in the third panel of Fig. 5 we obtain $\log \bar{k}_{2 \text { theo }}=-2.11 \pm 0.06$ at an age of $24.2 \times 10^{6}$ years, which is also in agreement with observations. The results concerning radii, effective temperature ratio, apsidal-motion rate and lithium depletion are very similar regardless of whether the models were computed without rotation, with solid rotation or local conservation of the angular momentum. This is due to the fact that the rotating models are not considerably perturbed by rotation in this case. Probably, the best results would be obtained from an intermediary formulation, as for example the integration the differential equation of the transport of angular momentum. However, case 2 can be used to verify the values obtained by Yildiz (2003) with respect to the ratio of core/envelope rotation rate. Our results differ mainly in two aspects: first, there is no discontinuities in the angular velocities inside any model and second, we have not detected differences as large as 65 times between the envelope and the nucleus. The ratio of angular rotation is always smaller (one order of magnitude) than this. If one uses the fourth order differential equation governing the transport of angular momentum coupled with the diffusion one, the ratio is still smaller than found in case 2 .

As previously commented, Yildiz (2003) finds an acceptable solution only if the core - containing $52 \%$ of the total masses - rotates 65 times faster than the outer layers. The question is: is such a redistribution of angular velocity possible for EK CepA, which is a very young star? Had this component enough time to achieve such a fast core (that does not coincide necessarily with its smaller convective core)? The only observational evidence of stellar internal rotation comes from the
Sun (see for example Schou et al. 1998; Chaplin et al. 1999; for a more extensive and updated review see Thompson et al. 2003). The distribution of angular velocities obtained from helioseismologic studies indicates that there are no large differences between surface and interior. Of course, there is a latitude dependence but the maximum ratio is around 1.4 times (Fig. 7 by Thomson et al. 2003). Theoretical calculations also reinforce the scenario of a rather flat distribution of angular velocities, as we have seen. Therefore, the angular velocity distribution assumed by Yildiz (nucleus rotating 65 times faster than the surface) seems to be not supported by observational and theoretical evidence. A fast rotating core, but smaller than $52 \%$ of the total mass, may be present in the primary only for much more evolved models.

The profile of internal rotation postulated by Yildiz also yields some difficulties. In the boundary core-envelope the drastic change of the angular velocity $\left(1.3 \times 10^{-3} \mathrm{rad} \mathrm{s}^{-1}\right.$ to $2.0 \times 10^{-5} \mathrm{rad} \mathrm{s}^{-1}$ ) may introduce some kind of instability.

In addition, a fast rotating nucleus would change the dynamics of the system, altering the rate of apsidal-motion. Indeed, Dicke (1970) invoked such a possibility to explain the advance of the perihelion of Mercury as due to the presence of a fast rotating core in the Sun.

\section{Future perspectives}

Although we are able to model EK Cep without the need for a rapid rotating core, some uncertainties still remain and we do not consider the solutions presented here as definitive ones. The aim of the present paper is to show that standard stellar models (or rotating ones without the need for a very fast rotating core) are capable of fitting the observational properties of EK Cep, including radius, masses, effective temperature ratio and apsidal-motion rate. We estimate that the accuracy of our solution is around $5-10 \%$.

The system should be reobserved both photometrically (preferently with Strömgren filters) and spectroscopically in order to reduce the observational uncertainties. But the theoretical aspects should also be worked out. Neverthless, large discrepancies between observations and theoretical prediction are found in double-lined eclipsing binaries whith a low mass ratio. This suggests that, apart from the intrinsic difficulties in determining the individual absolute dimensions for the less luminous component, some kind of physical process may occur during the formation and evolution of the system. A key question arises: is the chemical composition equally distributed between both components, as usually assumed? The distribution of light elements in the solar system indicates that there segregation between massive and not massive bodies. Can a similar physical process act also in the case of eclipsing binaries with low mass ratio? This possibility should be investigated more closely.

Acknowledgements. The author thanks J. Fernandes, I. Ribas and L. F. Miranda for many useful comments which helped to improve the manuscript. The Spanish MCYT (AYA2003-04651) is gratefully acknowledged for its support during this work. 


\section{References}

Andersen, J. 1991, A\&ARv, 3, 91

Alexander, D. R., \& Ferguson, J. W. 1994, ApJ, 437, 879

Alves, D. R. 2004, New Astron. Rev., 48, 659

Caton, D. B., \& Burns, W. C. 1993, IBVS No. 3900

Caton, D. B., Hawkins, R. L., \& Burns, W. C. 1989, IBVS No. 3408

Chaplin, W. J., Christensen-Dalsgaard, J., Elsworth, Y., et al. 1999, MNRAS, 308, 405

Claret, A., Giménez, A., \& Martín, E. L. 1995, A\&A, 302, 741

Claret, A. 1999, A\&A, 350, 56

Claret, A. 2004, A\&A, 424, 919

Claret, A., \& Giménez, A. 1989, A\&AS, 81, 1

Claret, A., \& Willems, B. 2002, A\&A, 388, 518

Dicke, R. H. 1970, ARA\&A, 8, 297

Diethelm, R. 1992, Bull. BBSAG, 89

Diethelm, R. 1993, IBVS, No. 3903

Endal, A. S., \& Sofia, S. 1976, ApJ, 210, 184

Giménez, A., \& Margrave, T. E. 1985, AJ, 90, 358

Giménez, A., \& Bastero, M. 1995, Ap\&SS, 226, 99

Hill, G., \& Ebbighausen, E. G. 1984, AJ, 89, 1256

Iglesias, C. A., \& Rogers, F. J. 1996, ApJ, 464, 943
Khaliullin, Kh. F. 1983, Sov. Astron., 27, 43

Kippenhahn, R., Meyer-Hofmesiter, E., \& Thomas, H. C. 1970, A\&A, 5,155

Kippenhahn, R., \& Thomas, R. C. 1970, in Stellar Rotation, ed. A. Slettebak (Dordrecht, Holland: D. Reidel, Publ. Co.), 20

Kopal, Z. 1959, Close Binary Systems (London: Chapman \& Hall)

Kopal, Z. 1978, Dynamics of Close Binary System (Dordrecht/ Holland: Reidel)

Lacy, C. H. S., \& Fox, G. 1994, IBVS, No. 4009

Levi-Civita, T. 1937, Amer. J. Math., 59, 225

Marques, J. P., Fernandes, J., \& Monteiro, M. J. P. F. G. 2004, A\&A, 422, 239

Martín, E. L., \& Rebolo, R. 1993, A\&A, 274, 274

Pajdosz, G. 1993, private communication

Popper, D. M. 1987, ApJ, 313, L81

Ribas, I., Giménez, A., Torra, J., Jordi, C., \& Oblak, E. 1998, A\&A, 330,600

Schou, J., et al. 1998, ApJ, 505, 390

Thompson, M. J., Christensen-Dalsgaard, J., Miesch, M. S., \& Toomre, J. 2003, ARA\&A, 41, 599

Yildiz, M. 2003, A\&A, 409, 689

Zahn, J.-P. 1992, A\&A, 265, 115 